This article is licensed under the Creative Commons Attribution-NonCommercial 4.0 International License (CC BY-NC) (http://www.karger.com/Services/OpenAccessLicense). Usage and distribution for commercial purposes requires written permission.

\title{
Differential Impact of Cognitive Impairment in MCl Patients: A Case-Based Report
}

\author{
Valentina Pergher ${ }^{a, b} \quad$ Birgitte Schoenmakers $^{c} \quad$ Philippe Demaerel $^{d}$ \\ Jos Tournoy ${ }^{e}$ f Marc M. Van Hulle \\ aDepartment of Cognitive Neuropsychology, Harvard University, Cambridge, MA, USA; \\ bLaboratory for Neuro- and Psychophysiology, Department of Neurosciences, KU \\ Leuven - University of Leuven, Leuven, Belgium; 'Academic Centre of General Practice, \\ KU Leuven - University of Leuven, Leuven, Belgium; dDepartment of Neuroradiology, KU \\ Leuven - University Hospitals Leuven, Leuven, Belgium; 'Department of Chronic Diseases, \\ Metabolism and Ageing, KU Leuven - University Hospitals Leuven, Leuven, Belgium; \\ fDepartment of Geriatric Medicine, KU Leuven - University Hospitals Leuven, \\ Leuven, Belgium
}

\section{Keywords}

Reserve $\cdot$ Mild cognitive Impairment $\cdot$ White matter $\cdot$ Grey matter $\cdot$ Neuropsychological tests

\section{Abstract}

Mild cognitive impairment $(\mathrm{MCl})$ traditionally refers to an intermediate stage between healthy individuals and early Alzheimer disease. Evidence shows grey and white matter volume changes and decrease in several executive functions, albeit the relation between cognitive performance and brain volume remains unclear. Here, we discuss 3 individual cases of $\mathrm{MCl}$ by investigating their MRI scans and cognitive test performance. We also recruited age-matched healthy older adults serving as gold standard for both grey and white matter volume and cognitive test outcomes. Our results show the impact of cognitive impairment on cognitive test performance and grey and white matter volumes, and the role played by cognitive and brain 


\section{Case Reports in Gastroenterology}

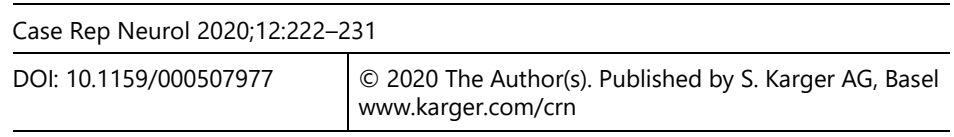

Pergher et al.: Impact of Cognitive Impairment on MCI Patients

reserve on mitigating cognitive decline. Furthermore, we add evidence to previous studies by showing an increase in white matter volume compared to healthy controls, in all 3 patients. This pattern of increased white matter volume might help us to better understand the pathological mechanisms underlying $\mathrm{MCl}$ which in turn could contribute to future investigations.

(C) 2020 The Author(s)

Published by S. Karger AG, Basel

\section{Introduction}

The transitional period leading up to the diagnosis of clinically probable early Alzheimer disease (AD) includes stages such as mild cognitive impairment (MCI), in which patients display signs of cognitive decline but their activities of daily living are largely preserved. Every year, 10-15\% MCI patients start to experience the first clinical symptoms of cognitive decline. However, the etiology of MCI is still not yet completely understood [1].

Grey matter loss has been reported for MCI patients in the hippocampus, medial temporal lobe, prefrontal cortex and insula, whereas white matter reduction in the temporal lobe, right anterior cingulate, frontal gyrus, and parietal angular gyrus [2]. Most of the MCI individuals exhibit atrophy patterns similar to AD patients, whereas for others they are similar to cognitively normal individuals [3]. Similarly, neuropsychological tests distinguish between normal aging and MCI, such as Digit Span Backward [4], Stroop task [5], and Mini-Mental State Examination (MMSE) [6].

Recent evidence shows that patient's occupation, leisure activities, and educational background might resort in a positive effect on one's mental abilities. They are considered part of cognitive reserve (CR), defined as the difference between one's actual and expected performance in cognitive tasks given a number of individual characteristics when present a brain pathology [7]. Two explanations have been put forward in the literature, passive and active. Brain reserve (BR) [8] belongs to the former as it assumes that larger brains tolerate better structural changes without displaying significant clinical deficits [9]. In contrast, active models suggest that CR helps to cope with brain damage by using pre-existing cognitive processes and compensatory processes [10]. In this way, individuals with higher CR are better equipped to withstand AD symptoms until a large amount of pathology has accumulated [7]. The effect of CR (education, leisure activities and IQ) has been shown also in MRI investigations and has been associated with grey matter volume alterations in the superior temporal gyrus and the left superior parietal lobule [11] and in the left caudate nucleus, thalamus and left post-central gyrus [12], whereas white matter volume changes have been observed in the hippocampus [13].

In 2017, several patients were diagnosed with MCI according to the National Institute on Aging (NIA) criteria [14] in the Memory Clinic at Gasthuisberg University Hospital, Leuven, Belgium. Three patients were selected for our case reports study (see online suppl. material for further details about inclusion and exclusion criteria and Table S1 for their demographics; for all online suppl. material, see www.karger.com/doi/10.1159/000507977). The aim of our study is to shed light on the relation between cognitive impairment - considering differences in age and education (CR), and brain size (BR) - grey and white matter volume, and behavioral outcomes. 


\section{Case Reports in Gastroenterology}

\begin{tabular}{l|l}
\hline Case Rep Neurol 2020;12:222-231 \\
\hline DOI: 10.1159/000507977 & $\begin{array}{l}\text { (c) 2020 The Author(s). Published by S. Karger AG, Basel } \\
\text { www.karger.com/crn }\end{array}$ \\
\hline
\end{tabular}

Pergher et al.: Impact of Cognitive Impairment on $\mathrm{MCI}$ Patients

\section{Case Reports}

We performed an MRI scan for each individual (for details, see online suppl. material) to detect grey and white matter volumes, and we investigated their percentile, defined as the value below/above which a given percentage of observations in a group of observations falls [15]. In order to be able to compare our MCI patients with healthy controls, we recruited 33 healthy older adults from our university's Academic Center for General Practice, matched for age, sex, and years of education and had them undergo the same battery of cognitive tests and 12 of them take an MRI scan. MR images were evaluated by a neuroradiologist for Global Cortical Atrophy (GCA), Koedam, Medial Temporal Atrophy (MTA), and Fazekas (see [12] for further details). Considering the outcomes of those visual rating scales, consequently we investigated the inter-regional grey and white matter volumes of specific regions of interest (ROIs). Last, a battery of neuropsychological tests was administered to participants, including: MMSE, Digit Span, Controlled Oral Word Association Test, Visual Association Test (VAT), Stroop, Visual Search, Raven, CORSI, and N-Back (see [12] for further details). Of these, we calculated the percentile by comparing the performance of each patient with the healthy controls $(n=33)$.

Patient 1 is an individual between 70 and 75 years old first presented at our memory clinic in April 2016 because of a mild forgetfulness mainly noticed by the daughter over the last 2 years. The patient's medical history contained an appendectomy in 1960, a fall with skull fracture in 2003 with good recovery and arterial hypertension for which the patient took lisinopril and hydrochlorothiazide. In addition, it has been reported that the patient has difficulties with orientation in new environments and also with the use of some household appliances. The patient is a former banking consultant holding a $\mathrm{PhD}$ degree, now retired, living at home with his wife. They often go out for walking or biking, and they attend to their garden. They often take care of their grandchildren and are also active in local activities for older people. The results from the neuropsychological evaluation reported a severe short-term memory impairment (VAT), short-term working memory (Digit Span), visuo-spatial short-term working memory (CORSI), general cognitive functioning (MMSE) and verbal fluency (COWAT). Slightly differently, attention and inhibitory control (Stroop) revealed a moderate impairment, whereas working memory (N-Back) and fluid intelligence (Raven) were reported in the average of healthy controls (Table 1). An MRI and subsequent FDG PET scan were performed, and a diagnosis of non-amnestic MCI was established. Patient 1 showed a minimal widening of the cortical sulci, more pronounced in the parietal cortex (precuneus), together with an unusual high signal in the left hippocampus/amygdala, possible normal, but other etiologies, e.g. auto-immune pathology, cannot be excluded. Furthermore, we observed a moderate enlargement of the lateral and third ventricle and of the left temporal horn, and only one small vascular lesion in the frontal periventricular white matter on the right side (Table 2). Consequently, after segmentation, we detected differences in both total intracranial volume (TIV) of grey and white matter compared to healthy controls (Fig. 1): the TIV of grey matter was $53.56 \%$, scoring in the 8th percentile, and of white matter was $67.16 \%$, scoring in the $92 \mathrm{nd}$ percentile. Furthermore, considering the outcomes of the visual rating scale evaluation, we investigated the inter-regional grey and white matter volumes of specific ROIs (Fig. 2 and online suppl. Table S2), and we found differences in grey matter volume in the left amygdala, left hippocampus, left SPL (area 5 and 7), right SPL (area 5 and 7), and for white matter volume 


\section{Case Reports in Gastroenterology}

Case Rep Neurol 2020;12:222-231

DOI: 10.1159/000507977

(c) 2020 The Author(s). Published by S. Karger AG, Basel www.karger.com/crn

Pergher et al.: Impact of Cognitive Impairment on MCI Patients

in the left amygdala, left hippocampus, left SPL (area 5 and 7), and right SPL (area 5 and 7) compared to healthy individuals.

Patient 2 is an adult between 80 and 85 years old that was referred by the GP because of progressive memory problems. Patient's medical history mentioned stomach ulcers, arterial hypertension and a total hip replacement. The medication consisted of perindopril, calcium and vitamin D supplements, zoledronate, and estriol. The patient had progressive complaints of memory loss and subtle word finding difficulties over the last year. There was no impact on the level of functioning. The patient went to school until the age of 17 (secondary school) and is now retired as a factory worker. MMSE at presentation (2017) was 24/30. The results from the neuropsychological evaluation revealed a severe impairment in attention (Visual Search), visual short-term memory (VAT), verbal fluency (COWAT), attention and inhibitory control (Stroop), fluid intelligence (Raven), and general cognitive functioning (MMSE). Differently, short-term working memory revealed a moderate impairment (Digit Span) and visuo-spatial short-term working memory (CORSI). However, working memory (N-Back) indicated an average performance compared to the healthy controls (Table 1). A diagnosis of multidomain amnestic MCI was established, and an MRI was performed which showed alterations in both grey and white matter volumes (Table 2) in terms of global widening of the cortical sulci, moderate enlargement of the lateral and third ventricles, and several vascular white matter lesions in frontal and parietal periventricular and subcortical (left > right) white matter in both hemispheres. After segmentation, we detected differences in both TIV of grey and white matter compared to healthy controls (Fig. 1): the TIV of grey matter was $49.42 \%$, scoring in the 0 th percentile, and of white matter was $46.80 \%$, scoring in the 84 th percentile. Furthermore, considering the outcomes of the visual rating scale evaluation, we investigated the inter-regional grey and white matter volumes of specific ROIs (Fig. 2 and online suppl. Table S2), and we found differences in grey matter volume in the left SPL (area 5 and 7) and right SPL (area 5 and 7), and in white matter volume in the left SPL (area 5 and 7) and right SPL (area 5 and 7).

Patient 3 is an individual between 75 and 80 years old who was referred from our diabetics clinic since the patient could not independently manage the insulin therapy. The patient forgot to measure the glucose levels and/or to administer the insulin therapy. The patient indicated that they had become more forgetful over the last 6 months and had difficulties with the former scientific activities as an archeologist (e.g., the patient could not follow meetings anymore, experiences difficulties understanding scientific texts, etc.). The patient was trained as an electrician and retired as head of the safety and prevention department of a company. The patient always had a specific interest for archeology and obtained a PhD in archeology in 2005. Patient's history mentions rheumatoid arthritis, partial nephrectomy for a renal cell carcinoma with subsequent depression, benign prostate hypertrophy, chronic pain and spinal stenosis. The patient scored 29/30 on the MMSE. Patient's drug list contained acetaminophen, amlodipine, dutasteride and tamsulosin, transdermal fentanyl, losartan, pramipexol, simvastatin, and insulin. Following further investigation by using MRI and neuropsychological evaluation, led to the diagnosis of multidomain non-amnestic MCI (Table 1). The results from the neuropsychological evaluation revealed a severe impairment in attention and inhibitory control (Stroop) and attention (Visual Search). In contrast, short-term working memory revealed a moderate impairment (Digit Span), verbal fluency (COWAT), general cognitive functioning (MMSE), fluid intelligence (Raven), and visuo-spatial short-term working memory (CORSI). 


\section{Case Reports in Gastroenterology}

\begin{tabular}{l|l}
\hline Case Rep Neurol 2020;12:222-231 \\
\hline DOI: 10.1159/000507977 & $\begin{array}{l}\text { @ 2020 The Author(s). Published by S. Karger AG, Basel } \\
\text { www.karger.com/crn }\end{array}$ \\
\hline
\end{tabular}

Pergher et al.: Impact of Cognitive Impairment on MCI Patients

However, short-term memory (VAT and N-Back) revealed an average performance compared to healthy controls (Table 1). An MRI was performed, reporting alterations in both grey and white matter volumes (Table 2) in terms of global widening of the cortical sulci and moderate enlargement of the lateral and third ventricles, and bilateral multiple vascular periventricular and subcortical white matter lesion with beginning confluency of the lesions. After segmentation, we detected differences in both TIV of grey and white matter compared to healthy controls (Fig. 1): the TIV of grey matter was $42.21 \%$, scoring in the 0th percentile, and of white matter was $60.25 \%$, scoring in the 92 nd percentile. Furthermore, considering the outcomes of the visual rating scale evaluation, we investigated the inter-regional grey and white matter volumes of specific ROIs (Fig. 2 and online suppl. Table S2), and we found differences in grey matter volume in the left SPL (area 5 and 7) and right SPL (area 5 and 7), and in white matter volume in the left SPL (area 5 and 7) and right SPL (area 5 and 7).

\section{Discussion}

These case reports provided a valuable addition to the current workup on MCI and its diagnosis, especially for the evidence of an increase in white matter volume. This study highlights increased white matter volume and decreased grey matter volume in MCI patients compared to healthy controls, although individual discrepancies in cognitive test performance were detected, suggesting that both CR [10] and brain size [8] played an important role in compensating for cognitive impairment.

In most MCI and AD literature studies, a white matter volume reduction is reported in several brain regions, such as precentral gyrus and superior frontal gyrus [16], following the retrogenesis theory of white matter degeneration [17], where the main feature is the loss or disconnection of fiber tracts. However, in contrast, our results revealed a larger white matter volume in all $3 \mathrm{MCI}$ patients compared to healthy controls when looking at the whole brain, whereas in 2 out of 3 patients when looking at specific ROIs (SPL right and left, areas 5 and 7). An increase in white matter volume has been reported for developing brains from childhood to adolescence [18] but not for MCI. Although the reason behind the observed increase is elusive to us, it might influence clinical outcome and diagnosis. As for grey matter volume, it displayed a decrease in all $3 \mathrm{MCI}$ patients when investigating the whole brain, and in 2 out of 3 patients when investigating specific ROIs (amygdala right and left, and SPL right and left, respectively for areas 5 and 7), in line with previous studies.

Furthermore, we measured cognitive functioning by using several cognitive tests. Patient 1 exhibited a smaller cognitive impairment, in line with the CR theory of Stern [10] as he/she was the youngest with a high education level and in line with the BR theory of Schofield [8] as he/she had the largest grey matter volume of all 3 patients. According to Schofield, individuals with larger brain size exhibit a smaller amount of cognitive impairment until a later stage due to the neural substrate support in maintaining a normal cognitive functioning. These findings are confirmed by studies that investigated the impact of CR and BR on cognitive decline [12]. In contrast to the first case, patient 2 was characterized by low educational level, older age and small brain size that affected negatively on neuropsychological performance and confirmed the role played from CR and BR on cognitive functioning. Differently, patient 3 revealed a severe impairment in certain cognitive functions, probably due to the negative effect played 


\section{Case Reports in Gastroenterology}

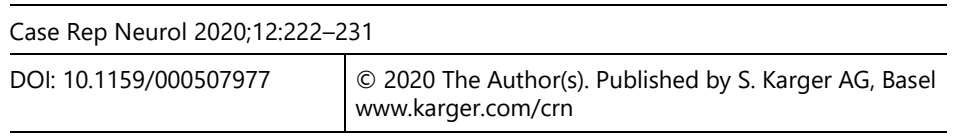

Pergher et al.: Impact of Cognitive Impairment on MCI Patients

by age, accordingly to the study of Greenwood [19], whereas a moderate impairment in other cognitive domains probably due to the high level of education. Also in this case, both CR and BR were involved in modulating the impact of cognitive impairment.

Neuroimaging studies investigating MCI patients reported grey matter volume decrease in the hippocampus, medial temporal lobe, prefrontal cortex, and insula [3], whereas white matter reduction in the temporal lobe, right anterior cingulate, frontal gyrus, and parietal angular gyrus [2]. In addition to these studies, our findings showed a grey matter volume decrease in amygdala and SPL that have only been sparsely investigated (SPL by [20,21], and amygdala by [22]), and reported evidence of an increase in white matter volume, previously not observed in MCI patients, which could play a critical role in the diagnosis of MCI. A possible cause of WM volume increase might be due to the fact that with aging and neurodegenerative diseases, there is an increase in neuronal inflammation [23] and an increase in the number of HLA-DR/MHC II-positive microglia [24].

In conclusion, we demonstrate the differential impact of CR and BR on cognitive impairment for structural and cognitive responses in MCI patients, and we underline the additional evidence compared to the previous studies regarding the increase in white matter volume in these patients that might reach a valuable level of importance in clinical practice. However, it is still not clear which factors may have contributed to the latter results and what their role is in predicting cognitive impairment. As limitations of our study, we mention the use of age and education as factors that might play an important role in cognitive performance and structural changes, although others, such as leisure activities, professional attainment, life style and sex $[7,25]$ can have a considerable impact as well.

\section{Acknowledgement}

We thank Prosper Agbesi Fiave for his constructive suggestions how to process the MRI scans.

\section{Statement of Ethics}

Prior to participation on this study, all participants read and, when they agreed, signed the informed consent, including publication agreement, that was approved by our Ethical Committee (UZ KU Leuven, S59475) and in accordance with the latest version of the Declaration of Helsinki.

\section{Disclosure Statement}

The authors declare that the research was conducted in the absence of any commercial or financial relationship that could be regarded as a potential conflict of interest. 


\section{Case Reports in Gastroenterology}

\section{Funding Sources}

V.P. was supported by a special research fund of the KU Leuven (C24/18/098), and M.M.V.H. by the European Union's Horizon 2020 research and innovation programme under grant agreement No. 857375, the Financing Program (PFV/10/008) and the special research fund of the KU Leuven (C24/18/098), the Belgian Fund for Scientific Research Flanders (G088314N, G0A0914N, G0A4118N), the Interuniversity Attraction Poles Programme - Belgian Science Policy (IUAP P7/11), and the Hercules Foundation (AKUL 043).

\section{Author Contributions}

V.P. and M.M.V.H. designed the study. V.P. and J.T. acquired the data. P.D. clinically evaluated the MRI scans. J.T. and B.S. assisted with participant recruitment. All authors contributed to data interpretation and to the writing of the article.

\section{References}

1 Petersen RC, Stevens JC, Ganguli M, Tangalos EG, Cummings JL, DeKosky ST. Practice parameter: early detection of dementia: mild cognitive impairment (an evidence-based review). Report of the Quality Standards Subcommittee of the American Academy of Neurology. Neurology. 2001 May;56(9):1133-42.

2 Wang Z, Guo X, Qi Z, Yao L, Li K. Whole-brain voxel-based morphometry of white matter in mild cognitive impairment. Eur J Radiol. 2010 Aug;75(2):129-33.

3 Fan Y, Batmanghelich N, Clark CM, Davatzikos C; Alzheimer's Disease Neuroimaging Initiative. Spatial patterns of brain atrophy in MCI patients, identified via high-dimensional pattern classification, predict subsequent cognitive decline. Neuroimage. 2008 Feb;39(4):1731-43.

4 Kurt P, Yener G, Oguz M. Impaired digit span can predict further cognitive decline in older people with subjective memory complaint: a preliminary result. Aging Ment Health. 2011 Apr;15(3):364-9.

5 Bélanger S, Belleville S, Gauthier S. Inhibition impairments in Alzheimer's disease, mild cognitive impairment and healthy aging: effect of congruency proportion in a Stroop task. Neuropsychologia. 2010 Jan;48(2):581-90.

6 Franzmeier N, Buerger K, Teipel S, Stern Y, Dichgans M, Ewers M; Alzheimer's Disease Neuroimaging Initiative (ADNI). Cognitive reserve moderates the association between functional network anti-correlations and memory in MCI. Neurobiol Aging. 2017 Feb;50:152-62.

7 Stern Y. Cognitive reserve. Neuropsychologia. 2009 Aug;47(10):2015-28.

8 Schofield P. Alzheimer's disease and brain reserve. Australas J Ageing. 1999;18(1):10-4.

9 Valenzuela MJ, Sachdev P. Brain reserve and dementia: a systematic review. Psychol Med. 2006 Apr;36(4):441-54.

10 Stern Y. What is cognitive reserve? Theory and research application of the reserve concept. J Int Neuropsychol Soc. 2002 Mar;8(3):448-60.

11 Solé-Padullés C, Bartrés-Faz D, Junqué C, Vendrell P, Rami L, Clemente IC, et al. Brain structure and function related to cognitive reserve variables in normal aging, mild cognitive impairment and Alzheimer's disease. Neurobiol Aging. 2009 Jul;30(7):1114-24.

12 Pergher V, Demaerel P, Soenen O, Saarela C, Tournoy J, Schoenmakers B, et al. Identifying brain changes related to cognitive aging using VBM and visual rating scales. Neuroimage Clin. 2019;22:101697.

13 Burke SL, Hu T, Fava NM, Li T, Rodriguez MJ, Schuldiner KL, et al. Sex differences in the development of mild cognitive impairment and probable Alzheimer's disease as predicted by hippocampal volume or white matter hyperintensities. J Women Aging. 2019 Mar-Apr;31(2):140-64.

14 Jack CR Jr, Albert M, Knopman DS, McKhann GM, Sperling RA, Carillo M, et al. Introduction to revised criteria for the diagnosis of Alzheimer's disease: National Institute on Aging and the Alzheimer Association Workgroups. Alzheimers Dement. 2011;7:257. 


\section{Case Reports in Gastroenterology}

DOl: $10.1159 / 000507977$

(C) 2020 The Author(s). Published by S. Karger AG, Basel www.karger.com/crn

Pergher et al.: Impact of Cognitive Impairment on MCI Patients

15 Hyndman RJ, Fan Y. Sample quantiles in statistical packages. Am Stat. 1996;50:361-5.

16 Salat DH, Greve DN, Pacheco JL, Quinn BT, Helmer KG, Buckner RL, et al. Regional white matter volume differences in nondemented aging and Alzheimer's disease. Neuroimage. 2009 Feb;44(4):1247-58.

17 Stricker NH, Schweinsburg BC, Delano-Wood L, Wierenga CE, Bangen KJ, Haaland KY, et al. Decreased white matter integrity in late-myelinating fiber pathways in Alzheimer's disease supports retrogenesis. Neuroimage. 2009 Mar;45(1):10-6.

18 Guo X, Chen C, Chen K, Jin Z, Peng D, Yao L. Brain development in Chinese children and adolescents: a structural MRI study. Neuroreport. 2007 Jun;18(9):875-80.

19 Greenwood PM. The frontal aging hypothesis evaluated. J Int Neuropsychol Soc. 2000 Sep;6(6):705-26.

20 Giorgio A, Santelli L, Tomassini V, Bosnell R, Smith S, De Stefano N, et al. Age-related changes in grey and white matter structure throughout adulthood. Neuroimage. 2010 Jul;51(3):943-51.

21 Terribilli D, Schaufelberger MS, Duran FL, Zanetti MV, Curiati PK, Menezes PR, et al. Age-related gray matter volume changes in the brain during non-elderly adulthood. Neurobiol Aging. 2011 Feb;32(2):354-68.

22 Poulin SP, Dautoff R, Morris JC, Barrett LF, Dickerson BC; Alzheimer's Disease Neuroimaging Initiative. Amygdala atrophy is prominent in early Alzheimer's disease and relates to symptom severity. Psychiatry Res. 2011 Oct;194(1):7-13.

23 Sloane JA, Hollander W, Moss MB, Rosene DL, Abraham CR. Increased microglial activation and protein nitration in white matter of the aging monkey. Neurobiol Aging. 1999 Jul-Aug;20(4):395-405.

24 Sheffield LG, Berman NE. Microglial expression of MHC class II increases in normal aging of nonhuman primates. Neurobiol Aging. 1998 Jan-Feb;19(1):47-55.

25 Buckley RF, Mormino EC, Rabin JS, Hohman TJ, Landau S, Hanseeuw BJ, et al. Sex differences in the association of global amyloid and regional tau deposition measured by positron emission tomography in clinically normal older adults. JAMA Neurol. 2019 May;76(5):542-51.

Philippe Demaerel, Jos Tournoy, and Marc M. Van Hulle share the last senior authorship
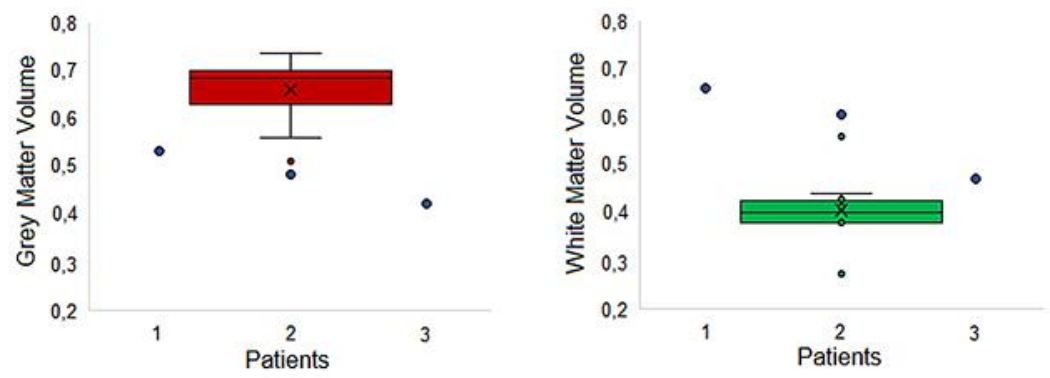

Fig. 1. Grey (left) and white (right) matter volume for $3 \mathrm{MCI}$ patients separately (black dots), compared with healthy controls (red and green box plots) $(n=12)$. Both red and green rectangles represent the interquartile range (IQR) of the healthy older adults, whereas the red and green dots represent the outliers, of the same population, below the "minimum" (Q1 - $1.5 \times$ IQR) and/or above the "maximum" (Q3 + $1.5 \times$ IQR) 


\section{Case Reports in Gastroenterology}
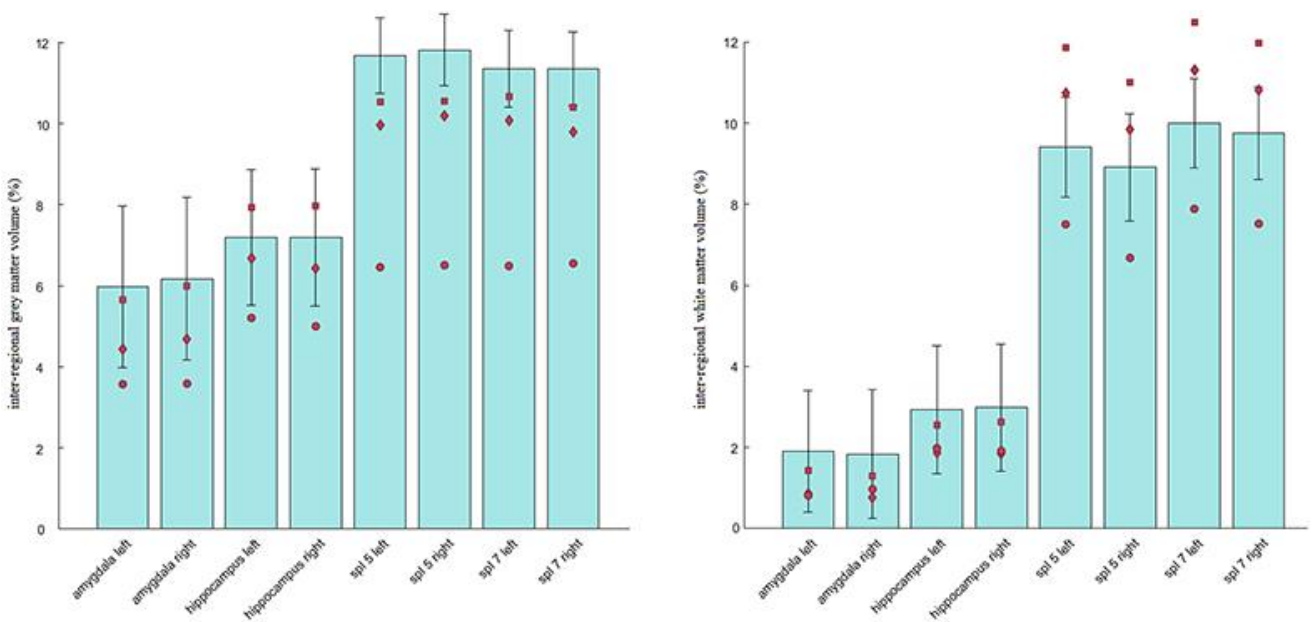

Fig. 2. Percent of grey (left) and white (right) matter volume for eight different ROIs of 3 MCI patients (square = patient 1 , diamond $=$ patient 2 , circle $=$ patient 3 ), compared with healthy controls (blue box plots $)(n=12)$.

Table 1. Cognitive tests of the three MCI patients and the matched healthy controls $(n=33)$ for each patient

\begin{tabular}{|c|c|c|c|c|}
\hline & Patient 1 & Patient 2 & Patient 3 & Healthy controls \\
\hline MMSE & 27/30 (3rd perc.) & 24/30 (0th perc.) & 28/30 (3rd perc.) & $29.51 \pm 0.83$ \\
\hline Digit Span (backward) & $2 / 10$ (0th perc.) & $5 / 10$ (12th perc.) & $5 / 10$ (12th perc.) & $6.45 \pm 2.13$ \\
\hline COWAT & $9 / 20$ (0th perc.) & $8 / 20$ (0th perc.) & $11 / 20$ (3rd perc.) & $15.22 \pm 4.41$ \\
\hline VAT & $1 / 6$ (0th perc.) & $0 / 6$ (0th perc.) & $6 / 6$ (51st perc.) & $5.03 \pm 1.26$ \\
\hline Stroop ${ }^{a}$ & 99/100 (3rd perc.) & $32 / 100$ (0th perc.) & $81 / 100$ (0th perc.) & $99.78 \pm 0.49$ \\
\hline CORSI & $4 / 10$ (0th perc.) & $3 / 10$ (8th perc.) & $4 / 10$ (8th perc.) & $7.31 \pm 1.77$ \\
\hline RAVEN & $38 / 60$ (24th perc.) & 2/60 (0th perc.) & $34 / 60$ (9th perc.) & $45.18 \pm 10.61$ \\
\hline Visual Search & $116 / 120$ (72nd perc.) & $99 / 120$ (0th perc.) & $10 / 120$ (0th perc.) & $112.18 \pm 4.75$ \\
\hline N-Back & $1 / 10$ (54th perc.) & $1 / 10$ (54th perc.) & $1 / 10$ (54th perc.) & $6.15 \pm 1.79$ \\
\hline
\end{tabular}

a More specifically, we used the third part of the test where inhibitory control is assessed in terms of accuracy level achieved in a fixed amount of time, not speed of processing. 
Case Reports in Gastroenterology
Case Rep Neurol 2020;12:222-231

\begin{tabular}{l|l}
\hline DOI: $10.1159 / 000507977$ & (C) 2020 The Author(s). Published by S. Karger AG, Basel
\end{tabular} www.karger.com/crn

Pergher et al.: Impact of Cognitive Impairment on $\mathrm{MCI}$ Patients

Table 2. Visual rating scale evaluation of the three MCI patients and the matched healthy controls $(n=33)$ for each patient

\begin{tabular}{lllll}
\hline & Subject 1 & Subject 2 & Subject 3 & Healthy controls \\
\hline GCA & 1 & 2 & 2 & $0.65 \pm 0.58$ \\
MTA right & 0 & 1 & 1 & $1.00 \pm 1.22$ \\
MTA left & 1 & 1 & 1 & $1.00 \pm 0.34$ \\
Koedam & 2 & 1 & 1 & $0.50 \pm 0.53$ \\
Fazekas & 0 & 1 & 2 & $1.00 \pm 0.74$ \\
\hline
\end{tabular}

